\title{
Determination of Advantages and Limitations of qPCR Duplexing in a Single Fluorescent Channel
}

\author{
Haoqing Zhang, Zhiqiang Yan, Xinlu Wang, Martina Gaňová, Honglong Chang, Soňa Lašsáková, \\ Marie Korabecna, and Pavel Neuzil*
}

Cite This: ACS Omega 2021, 6, 22292-22300

Read Online

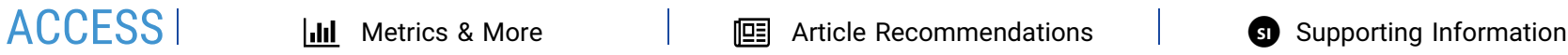

ABSTRACT: Real-time (quantitative) polymerase chain reaction (qPCR) has been widely applied in molecular diagnostics due to its immense sensitivity and specificity. qPCR multiplexing, based either on fluorescent probes or intercalating dyes, greatly expanded PCR capability due to the concurrent amplification of several deoxyribonucleic acid sequences. However, probe-based multiplexing requires multiple fluorescent channels, while intercalating dye-based multiplexing needs primers to be designed for amplicons having different melting temperatures. Here, we report a single fluorescent channel-based qPCR duplexing method on a model containing the sequence of chromosomes 21 (Chr21) and 18 (Chr18). We combined nonspecific intercalating dye EvaGreen with a 6-carboxyfluorescein (FAM) probe specific to either Chr21

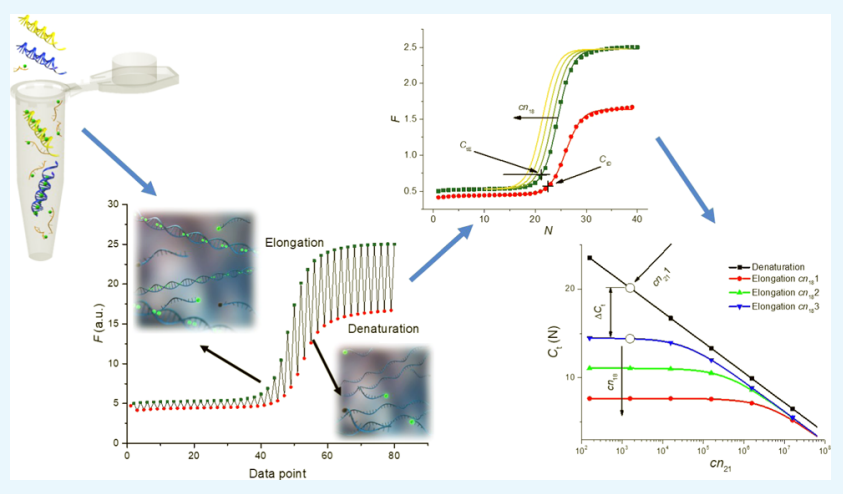
or Chr18. The copy number $(c n)$ of the target linked to the FAM probe could be determined in the entire tested range from the denaturation curve, while the $c n$ of the other one was determined from the difference between the denaturation and elongation curves. We recorded the amplitude of fluorescence at the end of denaturation and elongation steps, thus getting statistical data set to determine the limit of the proposed method in detail in terms of detectable concentration ratios of both targets. The proposed method eliminated the fluorescence overspilling that happened in probe-based qPCR multiplexing and determined the specificity of the PCR product via melting curve analysis. Additionally, we performed and verified our method using a commercial thermal cycler instead of a self-developed system, making it more generally applicable for researchers. This quantitative single-channel duplexing method is an economical substitute for a conventional rather expensive probe-based qPCR requiring different color probes and hardware capable of processing these fluorescent signals.

\section{INTRODUCTION}

Polymerase chain reaction (PCR) is a powerful technique to amplify a small number of specific sequences of deoxyribonucleic acid (DNA) molecules up to a detectable level. ${ }^{1}$ Since its invention in 1986, variants of PCR-based methods have been developed, ${ }^{2}$ such as standard PCR (end-point PCR), ${ }^{3}$ realtime/quantitative PCR (qPCR), ${ }^{4}$ and digital PCR (dPCR). ${ }^{5}$ qPCR is capable of monitoring the PCR progress in real-time and determining the initial concentration of the target genes. It is currently a gold standard for nucleic acid (NA) detection, especially during the coronavirus disease 2019 (COVID-19) pandemic. ${ }^{6}$ It is performed using either nonspecific intercalating fluorescent dyes, such as SYBR Green I or EvaGreen, ${ }^{7}$ or specific oligonucleotides (probes) with fluorophores, ${ }^{8}$ such as 6-carboxyfluorescein (FAM $)^{9}$ and $2^{\prime}$-chloro-7'phenyl-1,4-dichloro-6-carboxy-fluorescein (VIC). ${ }^{10}$

Multiplexing of any PCR expands its detection capability as more than one target gene is simultaneously amplified and detected. $^{11-14}$ qPCR multiplexing is often used for the detection of a DNA component with minor content with a background of another more abundant DNA, such as gene mutations with wild types in oncological research and diagnostics. $^{15,16}$ The infectious agents in the background of human sequences are often determined in microbiology and virology. ${ }^{17}$ The genes of genetically modified organisms are searched in food control. ${ }^{18}$ Paternally inherited diseaseassociated sequences of DNA are investigated in the background of cell-free DNA in the plasma of pregnant females, and they are used in noninvasive prenatal diagnostics. $^{19,20}$ The correct quantification of both mixture components by $\mathrm{qPCR}$ is complicated once there are large differences in their representation in the mixture.

Received: June 8, 2021

Accepted: August 2, 2021

Published: August 19, 2021 


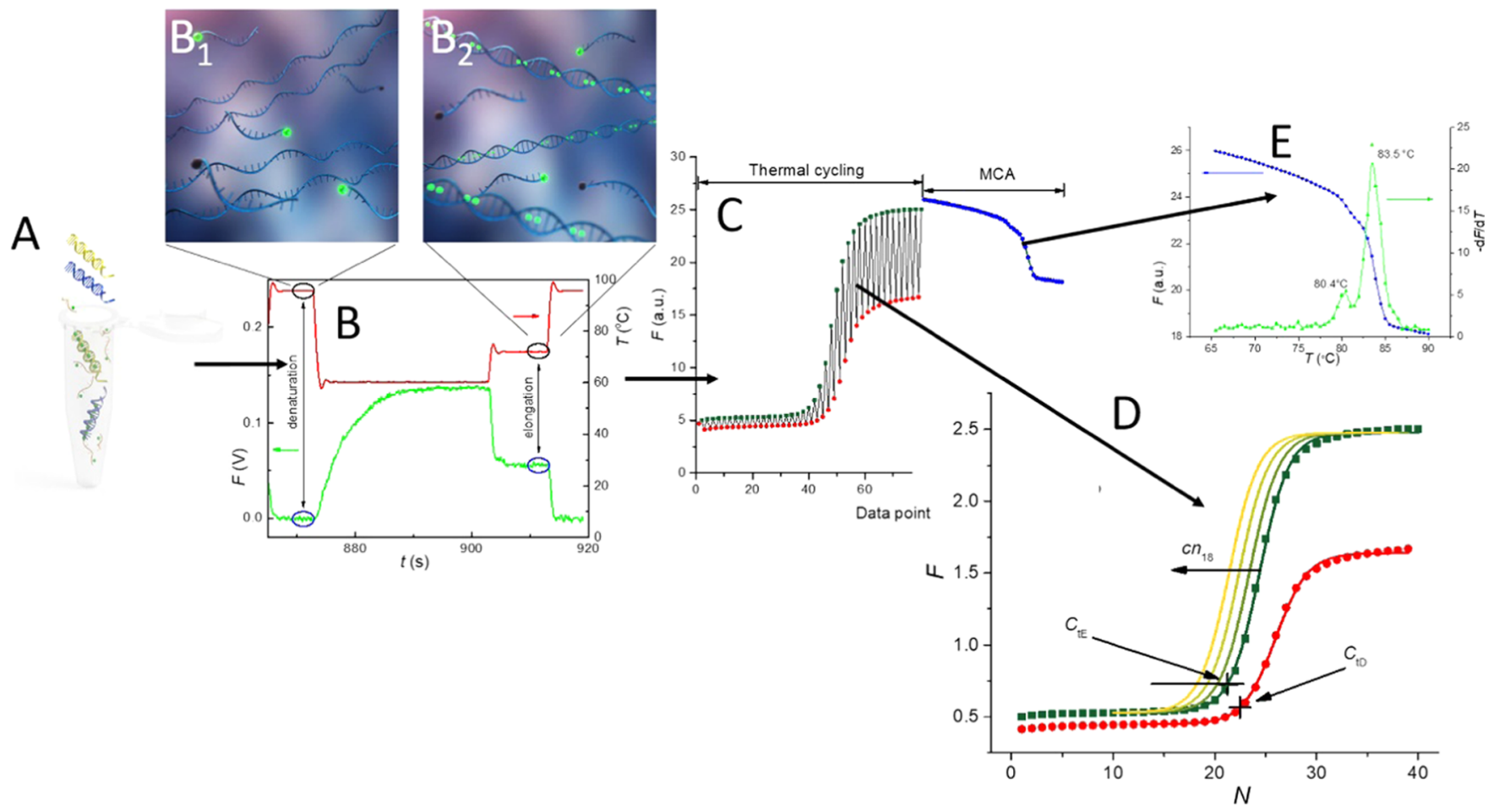

Figure 1. Principle of the proposed qPCR multiplexing method. (A) We prepared the PCR master mix containing two DNA templates, two sets of relevant forward and reverse primers, a FAM probe specific to one DNA template, and EvaGreen intercalating dye. (B) We performed the PCR and captured the $F$ (green curve) during each cycle at the end of the denaturation and elongation steps, while the red curve represents the heater temperature. Contributions to the $F$ at the denaturation step are only from the FAM probe (inset B1). $F$ at the elongation step consists of both the FAM probe linked to $\mathrm{Chr} 21$ as well as the EvaGreen intercalating dye with both targets (inset B2). (C) All of the collected data during the PCR followed by the MCA were split (D) into two sets and plotted separately, which represents the denaturation amplification curve (red) and the elongation amplification curves in gradient colors from dark to light green for different $c n_{18}$. (E) We also plotted an MCA as well as the negative derivation of $F$ showing the presence of two different amplicons in the PCR master mix.

Probe-based qPCR multiplexing utilizes probes attached to fluorophores with different emission wavelengths. The fluorescent signals are then split and detected using different optical channels. The number of target genes for the concurrent detection is theoretically unlimited as long as there is no emission wavelength overlap between the different probes. However, it is limited by the optical detection system as the increasing number of detectable colors makes the system complicated and costly. Additionally, the design of the probes and primers for each target gene should also be done carefully otherwise the interaction between them inhibits the PCR multiplexing efficiency. Expression analysis using qPCR highly depends on the quantification of all of the simultaneously analyzed targets. It is assumed that qPCR multiplexed amplification does not alter each other's efficiency. ${ }^{21,22}$ The prerequisites for the successful interpretation of duplexed qPCR experiments were extensively studied, which resulted in a generally accepted methodology of $2^{-\Delta \Delta C t}$, which is considered as the gold standard for the relative quantification of mixtures. $^{23}$

An intercalating dye-based qPCR multiplexing method simplifies the optical detection system as only one fluorescent channel is required, and there is no need to design different probes. The genes are typically distinguished based on different melting temperatures $\left(T_{M}\right)$ during the melting curve analysis (MCA) after temperature cycling. ${ }^{24,25}$ However, it is an endpoint detection, and primers specific to each target gene must be designed to have a different $T_{\mathrm{M}}$. Alternatively, it can be performed using the continuous fluorescence monitoring
(CFM) method allowing monitoring the PCR progress and optimizing it. ${ }^{26}$ It also enables to perform dynamic MCA during the transition from the elongation to denaturation step and to construct the PCR amplification curves of $\mathrm{two}^{27}$ or three genes, ${ }^{28}$ or perhaps more. Another CFM-based method was proposed combining the FAM probe with the EvaGreen dye for simultaneous detection as both FAM and EvaGreen share the same emission wavelength, ${ }^{29}$ which effectively doubles the PCR throughput. Unfortunately, both of these CFM-based methods are restricted from being further applied as they only allow one experiment at a time and require a customized setup. Recently, a novel single fluorescent channelbased multiplexing method was proposed using intercalating dyes, and the result was processed based on multidimensional standard curves. ${ }^{30-32}$ Moreover, the data set was achieved by commercial qPCR instruments, and the absolute quantification was determined, thus extending the use of these devices.

In this work, we performed a comprehensive study of singlechannel multiplexing based on the combination of EvaGreen and a FAM probe. We used a commercial qPCR instrument with 96 wells, which allowed us to study multiplexing using robust, statistically significant data sets. We showed the strengths and weaknesses of the proposed method and also compared it with the dual-channel multiplexing method, thereby making this PCR duplexing universal. Although the proposed work is based on the same principle as our previous one, $^{29}$ we performed a quantitative study of the method, having statistically significant data set using a commercial thermocycler instead of a home-made system to determine the 


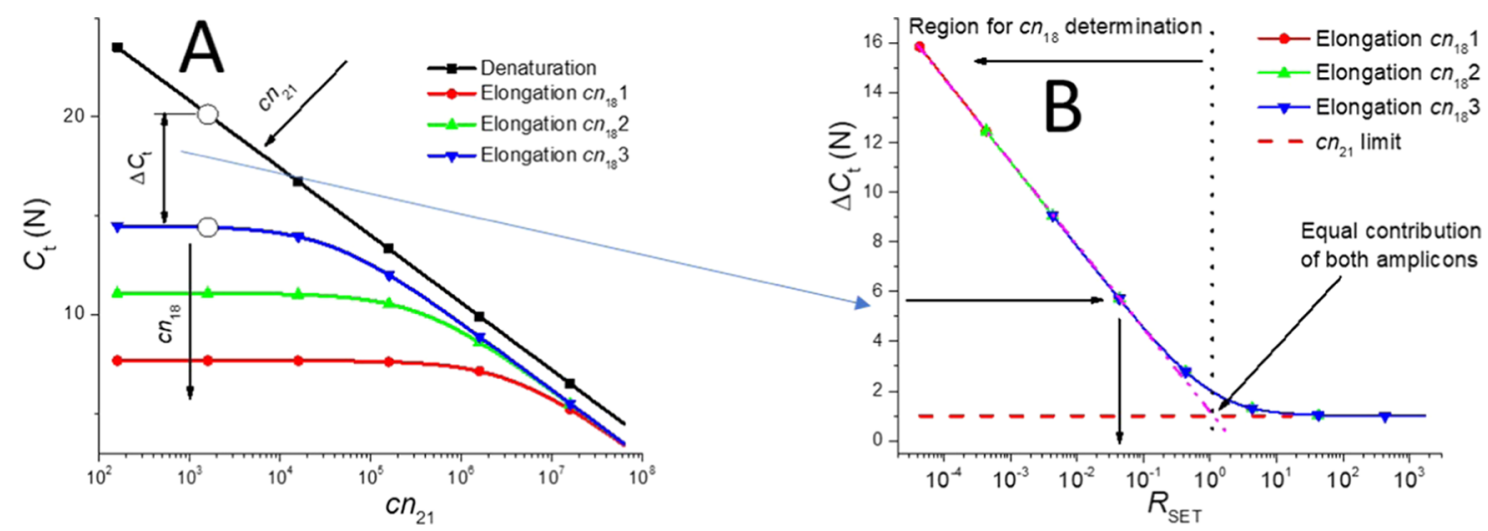

Figure 2. Determination of the copy numbers of both targets based on calculation. (A) Values of $C_{\mathrm{tD}}$ and $C_{\mathrm{tE}}$ as a function of $c n_{21}$ with $c n_{18}$ as the parameter. The black lines with squares are three denaturation curves overlapping each other that have been calculated using eq 1 . The blue curve with downward triangles, the green with upward triangles, and the red with circles are elongation curves calculated by eq 2. Each of them represents different values of $c n_{18}$. (B) $\Delta C_{t}$ values as a function of $R_{\mathrm{SET}}$ with $c n_{18}$ as the parameter that has been extracted from Figure $2 \mathrm{~A}$. All three curves overlap, which shows that it is the $R_{\mathrm{SET}}$ value determining the $\Delta C_{\mathrm{t}}$ instead of an absolute value of $c n_{18}$.

limit of this method in detail in terms of detectable concentration ratios of both targets. The proposed methodology was more generally applicable for system optimization compared with the previous one, as researchers could perform it using any commercial qPCR instruments. We show fully quantitative duplexing capable to be performed by a conventional thermocycler and thus available to anyone.

\section{PRINCIPLE OF THE PROPOSED MULTIPLEXING METHOD}

We assumed that we prepared PCR master mix using a fragment of chromosome $21(\mathrm{Chr} 21)$ specific to a FAM probe with a fixed number of copies $\left(c_{21}\right)$ and a fragment of chromosome 18 (Chr18) with a fixed number of copies $\left(c n_{18}\right)$ as the parameter (Figure 1A). We then performed the PCR in the presence of EvaGreen while collecting the fluorescent amplitude $(F)$ at the end of the denaturation as well as the elongation in each PCR cycle (Figure 1B), followed by an MCA (Figure 1C). We split the collected data and formed two amplification curves, the denaturation and elongation curves (Figure 1D). The denaturation curve only consists of the FAM probe as all of the double-stranded DNA molecules melted into single-stranded DNAs, and EvaGreen's contribution to $F$ is negligible. Changing $c n_{18}$ has no influence on the denaturation curve. The elongation curves represent EvaGreen intercalated with both $\mathrm{Chr} 21$ and $\mathrm{Chr} 18$ amplicons in combination with the FAM probe linked to Chr21. Changing $c n_{18}$ causes the PCR curve shift. The MCA extracted at the end of the PCR proved the presence of both amplicons in the PCR product (Figure 1E).

The threshold of the denaturation curve $\left(C_{\mathrm{tD}}\right)$ of the mixture is only determined by the value of $\mathrm{Cn}_{21}$ as the only FAM probe presence, and its concentration contributes to the $F$ value at $T_{\mathrm{D}}$ (Figure $2 \mathrm{~A}$, black line with squares)

$$
C_{\mathrm{tD}}=A_{\mathrm{D}}-B_{\mathrm{D}} \times \log \left(c n_{21}\right)
$$

where $A_{\mathrm{D}}$ is an offset and $B_{\mathrm{D}}$ is a PCR curve slope determined by the reaction efficiency of $\mathrm{Chr} 21$.

The threshold of the elongation curve $\left(C_{\mathrm{tE}}\right)$ is affected by both $c n_{18}$ and $c n_{21}$, and we can create a plot of $C_{\mathrm{tE}}$ as a function of $c n_{21}$ with $c n_{18}$ as the parameter

$$
C_{\mathrm{tE}}=A_{\mathrm{E}}-B_{\mathrm{E}} \times \log \left(c n_{18}+c n_{21}\right)
$$

where $A_{\mathrm{E}}$ is an offset and $B_{\mathrm{E}}$ is the overall PCR efficiency (Figure 2A, blue, red, and green lines). Equation 2 can be split into two limiting situations, $c n_{18} \gg c n_{21}$

$$
C_{\mathrm{tE} 18}=A_{1}+B_{1} \times \log \left(c n_{18}\right)
$$

and $c n_{18} \ll c n_{21}$

$$
C_{\mathrm{tE} 21}=A_{2}+B_{2} \times \log \left(c n_{21}\right)
$$

where $A_{1}$ and $A_{2}$ are the offsets and $B_{1}$ and $B_{2}$ are the individual PCR efficiencies of Chr18 and Chr21 amplifications, respectively.

We then extracted the differences from the corresponding $C_{t}$ values between the elongation and denaturation curves $\left(\Delta C_{t}\right)$ and plotted them as a function of the ratio between the $c n$ of the amplicon with and without a link to FAM $\left(R_{\mathrm{SET}}\right)$ with $\log c n_{18}$ as the parameter. Here, it would be $R_{\mathrm{SET}}=c n_{18} / c n_{21}$. It shows that the $\Delta C_{\mathrm{t}}$ value is a function of $R_{\mathrm{SET}}$ independent of the $c n$ absolute values (Figure $2 \mathrm{~B}$ ). The $\Delta C_{\mathrm{t}}$ can be expressed as

$$
\begin{aligned}
\Delta C_{\mathrm{t}} & =A_{\mathrm{D}}-A_{\mathrm{E}}+B \times\left[\log \left(c n_{21}\right)-\log \left(c n_{18}+c n_{21}\right)\right] \\
& =A+B \times \log \left[\frac{c n_{21}}{c n_{18}+c n_{21}}\right]
\end{aligned}
$$

where $A$ is intercept with the $Y$-axis and $B$ is a slope, respectively. We can then, with eq 4 , simplify the relationship as

$$
\begin{aligned}
\Delta C_{\mathrm{t}} & =A+B \times \log \left[\frac{c n_{21}}{c n_{21} R_{\mathrm{SET}}+c n_{21}}\right] \\
& =A+B \times \log \left[\frac{1}{R_{\mathrm{SET}}+1}\right]
\end{aligned}
$$

to show its independence on an absolute value of $\mathrm{cn}_{21}$ and $c n_{18}$ (Figure 2B). Parameter $B$ defines the precision of $R_{\mathrm{SET}}$ and thus $\mathrm{Cn}_{18}$ determination.

Thus, once we form the PCR standard curve, as shown in Figure 2A, we can determine the $\mathrm{cn}_{21}$ of an unknown sample from the denaturation curve (marked by a black arrow). Then, we calculate the $\Delta C_{\mathrm{t}}$ value and determine the $\mathrm{cn}_{18}$ based on the extracted $R_{\mathrm{SET}}$ values from Figure 2B. Note that the $\mathrm{cn}_{18}$ can only be determined from the left part of the graph starting at 

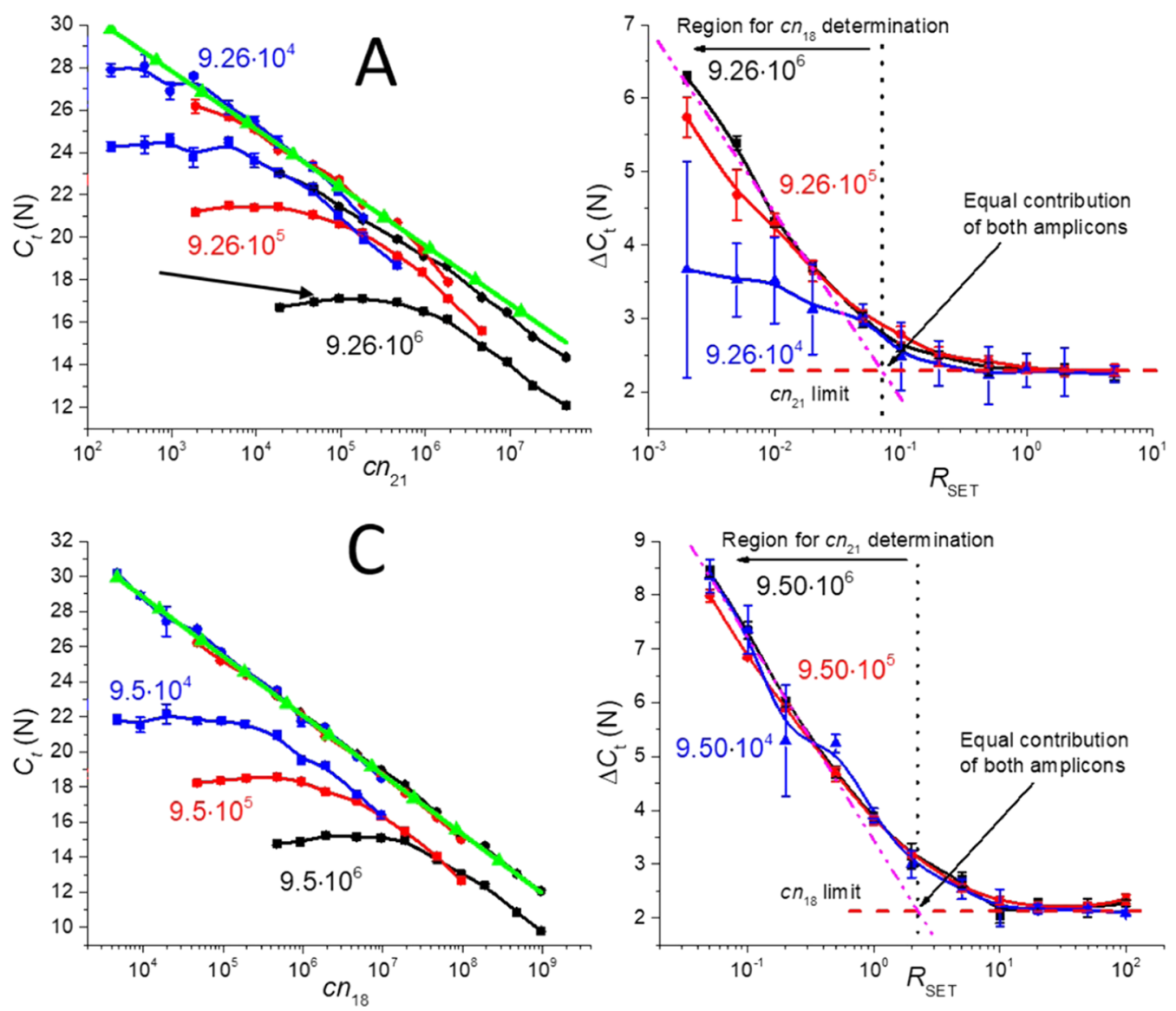

Figure 3. $C_{\mathrm{t}}$ values extracted from the PCR with EvaGreen intercalating dye and the FAM probe. (A) PCR standard curves of the variable $c n_{21}$ values with three different $c n_{18}$ values as the parameter with the denaturation curves marked with circles and the elongation with squares. The $c n_{21}$ values could be determined in an entire range from the denaturation standard curves. The arrow pointed to the curved region where minor Chr 21 clearly influences Chr18 amplification. The green line marked with triangles was formed by the curve fitting of an average value from all three denaturation curves. (B) Values of $\Delta C_{\mathrm{t}}$ plotted as a function of $R_{\mathrm{SET}}$ showing the importance of the $R_{\mathrm{SET}}$ value and not the individual $c n_{21}$ or $c n_{18}$. (C) Equivalent of (A) with $c n_{18}$ as the variable and $c n_{21}$ as the parameter with elongation curves marked by squares and the denaturation by circles. (D) Equivalent of (B) with $c n_{18}$ as the variable and $c n_{21}$ as a parameter.

the point where both amplicons' contribution is equal; otherwise, $\mathrm{cn}_{21}$ dominates the change of $\mathrm{C}_{\mathrm{tE}}$.

\section{RESULTS AND DISCUSSION}

3.1. Preliminary Experiments. We first performed the PCR and extracted elongation and denaturation standard curves of Chr21 with $\mathrm{cn}_{21}$ from $9.5 \times 10^{1}$ to $9.5 \times 10^{6}$ with and without a FAM probe to determine the $C_{\mathrm{t}}$ shift from the elongation to the denaturation as well as the PCR efficiency for both curves. Each experiment was repeated four times to suppress any random error. Then, the same experiment was conducted using $\mathrm{Chr} 18$ from $\mathrm{cn}_{18}$ between $9.26 \times 10^{2}$ and 9.26 $\times 10^{6}$ with a VIC probe (Supporting Information, Section S1). We used a VIC specific to Chr18 as a reference to the singlechannel multiplexing method. We found that the PCR efficiency was very similar for all combinations, and its value is $(-3.46 \pm 0.03) N \times(\log c n)^{-1}$ (mean \pm fitting error from four measurements), which demonstrated the correct experiment setting as well as the minimal pipetting error (Supporting Information, Section S1).

3.2. Multiplexing Using the Target $\mathrm{Cn}_{18}$ Constant and the $c n_{21}$ Variable with FAM Specific to Chr21. We then mixed Chr21 with a FAM probe and Chr18 with the ratio $R_{\text {SET }}$ with three different $c n_{18}$ values of $9.26 \times 10^{4}, 9.26 \times 10^{5}$, and
$9.26 \times 10^{6}$ and a $\mathrm{cn}_{21}$ variable (Supporting Information, Section S2). We performed the PCR and extracted the denaturation and elongation standard curves (Figure 3A). The denaturation curves (black, red, and blue line with the squares) were solely determined by $\mathrm{cn}_{21}$, but they were nevertheless influenced by the abundance of Chr18, as exhibited by the curve deviation from the straight line.

The elongation curves (black, blue, and red with circles) show two distinguished regions, one determined by $\mathrm{cn}_{18}$ and the other by $\mathrm{Cn}_{21}$, as predicted by eqs $2-6$, and their graphical representation is shown in Figure 2A. We performed the curve fitting (green line in Figure $3 \mathrm{~A}$ ) of the average of all three denaturation curves using eq 1 and obtained $A_{\mathrm{D}}$ and $B_{\mathrm{D}}$ as $(36.03 \pm 0.56) N$ and $(-2.74 \pm 0.11) N \times\left(\log c n_{21}\right)^{-1}$ (both mean \pm fitting error), respectively.

The saturation at the elongation curve determined by the $c n_{18}$ value exhibited a curved shape instead of a flat line parallel to the $X$-axis (pointed by an arrow in Figure 3A), a clear sign of $\mathrm{cn}_{21}$ influence. With a lower value of $c n_{21}$, the $C_{\mathrm{tE}}$ was also lowered, demonstrating that the influence of $\mathrm{Chr} 21$ was diminishing. All of the PCR experiments were accompanied with MCAs, and there was a trend showing the transition from the region dominated by $\mathrm{Chr} 21$ to a region dominated by Chr18 with an increased number of amplicons of Chr18, while 

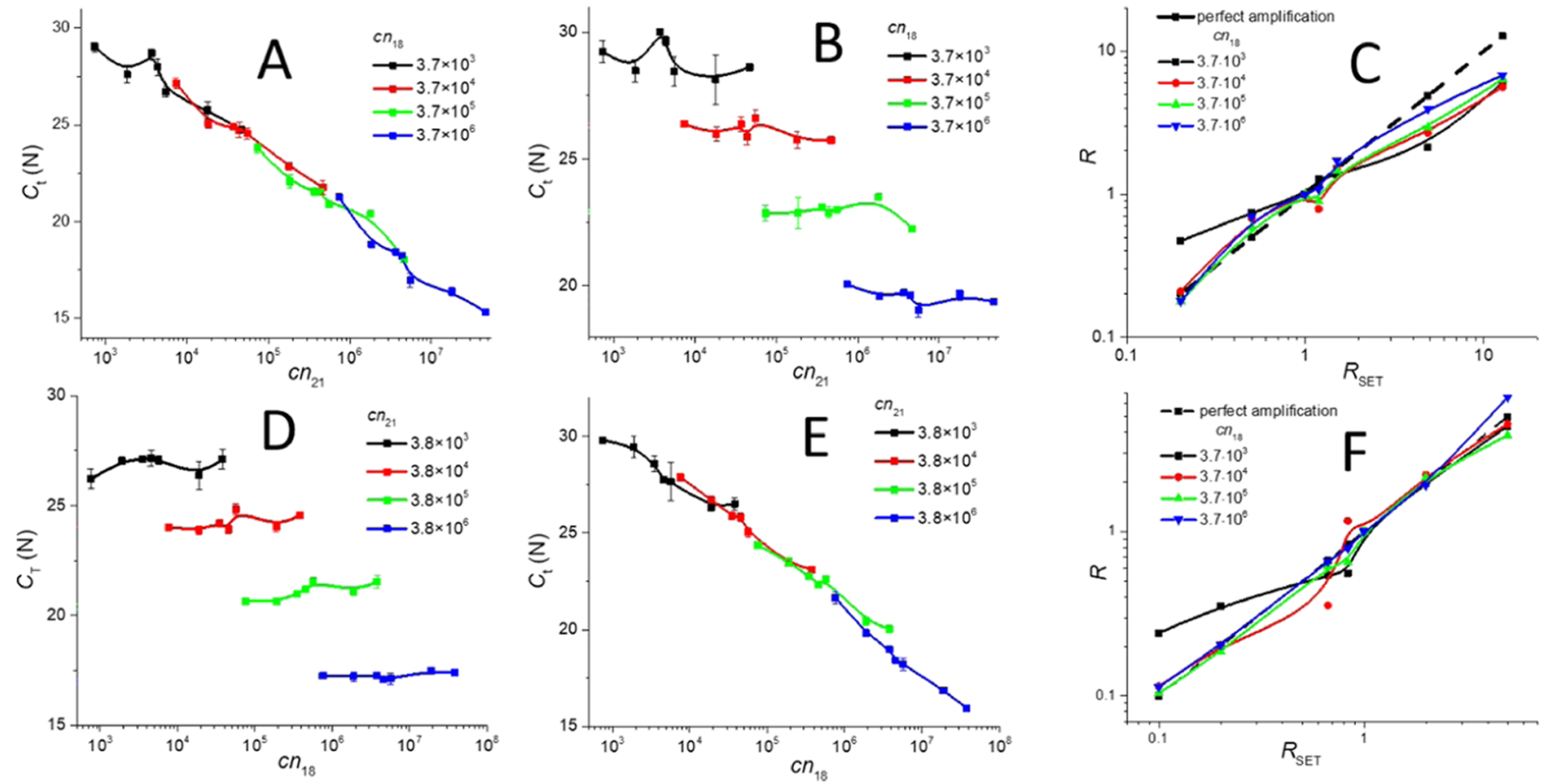

Figure 4. $C_{\mathrm{t}}$ values extracted from the PCR with FAM and VIC probes. (A) FAM channel with a $c n_{21}$ variable with $c n_{18}$ as the parameter showing the problems with the dual-channel amplification as the standard curves with different values of $c n_{18}$ should be collinear and form a single line regardless of the $c n_{18}$ value. (B) Same PCR mixtures using a VIC channel also showed the influence of $c n_{21}$ on $c n_{18}$ as the results should be independent of $\mathrm{Cn}_{21}$ since it was Chr18 with the VIC probe. (C) Implementation of the $2^{-\Delta \Delta C t}$ method showing its shortcoming with the noncompensated data for fluorescence spillover. (D-F) Similar results for a mixture with the $c n_{18}$ value variable and the $c n_{21}$ as the parameter.

the product of Chr21 dominated regardless of $\mathrm{cn}_{21}$ (Supporting Information, Section S3).

We then calculated the values of $\Delta C_{t}$ and plotted them as a function of $R_{\mathrm{SET}}$ (Figure $3 \mathrm{~B}$ ). We found that each amplicon had different contributions to the $C_{\mathrm{tE}}$ value as the point of the equal contribution of both the amplicons was not for $R_{\mathrm{SET}}=1$. We then modified the original elongation curves per eq 2 as

$$
C_{\mathrm{tE}}=A_{\mathrm{E}}-B_{\mathrm{E}} \times \log \left(c n_{18}+R_{\mathrm{C}} \times c n_{21}\right)
$$

where the critical ratio $\left(R_{\mathrm{C}}\right)$ was a coefficient reflecting the different contributions of each amplicon to the $C_{\mathrm{tE}}$ value. Then, eq 6 would convert to

$$
\begin{aligned}
\Delta C_{\mathrm{t}} & =A+B \times \log \left[\frac{c n_{21}}{c n_{18}+R_{\mathrm{C}} \times c n_{21}}\right] \\
& =A+B \times \log \left[\frac{R_{\mathrm{C}}}{R_{\mathrm{SET}}+R_{\mathrm{C}}}\right]
\end{aligned}
$$

We extracted the slope $B$ from the curves as $(-2.40 \pm 0.03) N$ $\times\left(\log R_{\mathrm{SET}}\right)^{-1}$ (mean \pm fitting error), thereby defining the sensitivity of $R_{\mathrm{SET}}$ determination as well as the $R_{\mathrm{C}}$ value of $\approx 0.07 R_{\mathrm{SET}}$. The low $R_{\mathrm{C}}$ value in comparison with $R_{\mathrm{SET}}$ showed strong dominance of $\mathrm{Chr} 21$ contribution to the $C_{\mathrm{tE}}$ values. As predicted by eq 7 , the $\Delta C_{\mathrm{t}}$ was a function of $R_{\mathrm{SET}}$ instead of an absolute number of either $c n_{21}$ or $c n_{18}$.

We determined the absolute value of $\mathrm{Cn}_{21}$ in the entire range from the denaturation curve (curves in Figure 3A marked with circles) with a precision of $(-2.74 \pm 0.11) N \times\left(\log c n_{21}\right)^{-1}$ (mean \pm fitting error). The $c n_{18}$ value was identified by extracting the $R_{\mathrm{SET}}$ value from Figure $3 \mathrm{~B}$ and multiplying it with $\mathrm{Cn}_{21}$.
We found that the precision of the $\mathrm{cn}_{18}$ determination is $(-2.40 \pm 0.03) N \times\left(\log R_{\mathrm{SET}}\right)^{-1}$ (mean \pm fitting error) for $R_{\mathrm{SET}}$ values smaller than $R_{\mathrm{C}} \approx 0.07$. The lower values of $c n_{18}$ deviated from the described behavior because the presence of Chr21 probably inhibited the PCR of Chr18, as shown by the MCAs (Supporting Information, Section S3).

3.3. Multiplexing Using a Target $\mathrm{Cn}_{21}$ Constant and a $\mathrm{cn}_{18}$ Variable with FAM Specific to Chr18. We repeated the same measurement with a FAM probe specific to Chr18 and Chr21 with $\mathrm{cn}_{21}$ fixed at the three different values with the $\mathrm{Cn}_{18}$ variable and plotted the results into Figure 3C (Supporting Information, Section S4). Here, the $R_{\mathrm{SET}}$ was $\mathrm{Cn}_{21} / \mathrm{cn}_{18}$. We performed the curve fitting as it was done in the previous section and found that the transition $R_{\mathrm{C}}$ changed from 0.07 to 2.26, which showed the dominance of the Chr21 regardless of the FAM probe specificity. We also calculated the $\Delta C_{\mathrm{t}}$ and plotted it as a function of $R_{\mathrm{SET}}$ (Figure $3 \mathrm{D}$ ). The results verified our original hypothesis that $\Delta C_{t}$ was determined by the value of $R_{\mathrm{SET}}$ instead of the absolute values of $\mathrm{Cn}_{18}$ and $\mathrm{cn}_{21}$.

The absolute value of $\mathrm{cn}_{18}$ couldbe determined in an entire range from the denaturation curve (curves in Figure 3C marked with squares) with a precision of $(-3.38 \pm 0.03) \mathrm{N} \times$ $\left(\log c n_{21}\right)^{-1}$ (mean \pm fitting error). The $\mathrm{cn}_{21}$ value could be extracted from Figure $3 \mathrm{D}$ using the $R_{\mathrm{SET}}$ values and a value of $c n_{18}$ with a precision of $(-3.48 \pm 0.26) N \times\left(\log R_{\mathrm{SET}}\right)^{-1}$ (mean \pm fitting error) for the $R_{\mathrm{SET}}$ values smaller than $R_{\mathrm{C}} \approx$ 2.26 .

3.4. Multiplexing with Target $c n_{21}$ Having a Chr21Specific FAM Probe and $\mathrm{Cn}_{18}$ with a Chr18-Specific VIC Probe. We performed a PCR using Chr21 and Chr18 specific to FAM and VIC probes, respectively, with either a $c n_{18}$ 
constant and a $\mathrm{cn}_{21}$ variable or vice versa (Supporting Information, Section S5).

Each experiment was conducted five times. We then extracted the $C_{\mathrm{t}}$ values using eq 11 and plotted $C_{\mathrm{t}}$ as a function of $\mathrm{Cn}_{21}$ from the FAM and VIC channels, as shown in Figure 4A,B, respectively.

We calculated the average errors in $C_{t}$ determination (Table 1 ), and they were rather small-between 2.4 and $1.5 \%$ for the

Table 1. Results from Two Fluorescence Channels Showing $C_{t}$ Determination Errors

\begin{tabular}{|c|c|c|c|c|}
\hline & $c n$ & intercept $(N)$ & slope $(\log c n)$ & $\begin{array}{c}\text { mean value } \\
(N)\end{array}$ \\
\hline \multicolumn{5}{|c|}{$c n_{21}$ variable } \\
\hline $\begin{array}{l}\text { FAM } \\
\text { channel }\end{array}$ & & $38.60 \pm 0.43$ & $-3.05 \pm 0.08$ & \\
\hline \multirow{4}{*}{$\begin{array}{l}\text { VIC } \\
\text { channel }\end{array}$} & $3.7 \times 10^{3}$ & & & $29.03 \pm 0.70$ \\
\hline & $3.7 \times 10^{4}$ & & & $26.13 \pm 0.33$ \\
\hline & $3.7 \times 10^{5}$ & & & $22.92 \pm 0.35$ \\
\hline & $3.7 \times 10^{6}$ & & & $19.55 \pm 0.30$ \\
\hline \multicolumn{5}{|c|}{$c n_{18}$ variable } \\
\hline \multirow{4}{*}{$\begin{array}{l}\text { FAM } \\
\text { channel }\end{array}$} & $3.8 \times 10^{3}$ & & & $26.89 \pm 0.37$ \\
\hline & $3.8 \times 10^{4}$ & & & $24.16 \pm 0.35$ \\
\hline & $3.8 \times 10^{5}$ & & & $20.99 \pm 0.42$ \\
\hline & $3.8 \times 10^{6}$ & & & $17.22 \pm 0.16$ \\
\hline $\begin{array}{l}\text { VIC } \\
\text { channel }\end{array}$ & & $39.59 \pm 0.39$ & $-3.07 \pm 0.07$ & \\
\hline
\end{tabular}

lowest and highest $c n_{18}$. The determination of the individual $C_{t}$ values varied much more, especially for the lowest value of $c n_{18}$ (black line in Figure 4B). We used the $2^{-\Delta \Delta C t}$ method $^{23}$ with the mixture having $R_{\mathrm{SET}}=1$ as the calibrator. We plotted the extracted $2^{-\Delta \Delta C t}$ values as a function of $R_{\mathrm{SET}}$ (Figure $4 \mathrm{C}$ ). The results showed that even the widely accepted method of $2^{-\Delta \Delta C t}$ had a problem with determining $R$ precisely when applied to the data without any compensation for fluorescence spillover. ${ }^{33}$

We repeated the same experiment with the $\mathrm{Cn}_{18}$ variable and $\mathrm{Cn}_{21}$ as the parameter and extracted the results in the same fashion as for the first experiment (Figure 4D-F), and the extracted data and errors were shown in Table 1.

3.5. Comparison of the Single-Channel Duplexing Method with the Method Employing Two Probes with Different Fluorophores. We performed the single-channel duplexing qPCR method using Chr21 with a FAM-specific probe and Chr18 both in the presence of EvaGreen intercalating dye. We also alternated the mixture composition having a FAM-specific probe to the Chr18 sequence instead of Chr21. We found that we could determine the value of the $\mathrm{cn}$ of the amplicon with the FAM probe in the entire range of the values. The second amplicon, cn, could only be determined once the $R_{\mathrm{SET}}$ value was smaller than the $R_{\mathrm{C}}$, which could vary for each individual pair of targets. In comparison, we performed standard multiplexing using FAM and VIC probes. The multichannel technique exhibited large error especially for the lower values of $c n s$, where the $C_{t}$ value varied in a range between 27 and 30 (black line in Figure 4B). We then processed the extracted data using the $2^{-\Delta \Delta C t}$ method $^{23}$ without performing channel overspilling compensation. It showed that even these widely accepted methods did not produce precise data (Figure 4C,F). Single-channel multiplexing produced more precise data, but it could determine both amplicons only for $R_{\mathrm{SET}}<R_{\mathrm{C}}$ in half of the total range of cn.

\section{CONCLUSIONS}

We proposed and verified a method of quantitative polymerase chain reaction (qPCR) duplexing using a single fluorescein isothiocyanate (FITC) fluorescent channel with a commercially available qPCR system with a 96-well platform. We amplified the following two sequences: one from chromosome 21 (Chr21) and one from chromosome 18 (Chr18) in the presence of the EvaGreen intercalating dye. We added a 6carboxyfluorescein (FAM) probe specific to either Chr21 or to Chr18 and extracted the PCR curves after the denaturation and elongation steps, and then, we plotted the PCR standard curves, one for denaturation and the second one for elongation. We were able to determine the copy number $(\mathrm{cn})$ of an amplicon with a FAM-specific probe in the entire range of the $c n$ values from the denaturation standard curve with precision values of $(-2.74 \pm 0.11) N \times\left(\log c n_{21}\right)^{-1}$ and $(-3.38 \pm 0.03) N \times\left(\log c n_{18}\right)^{-1}$ (both mean \pm fitting error) for Chr21 and Chr18, respectively.

The $c n$ value of the other amplicon was extracted from the difference of the critical thresholds between the denaturation and elongation curves with precision values of $(-2.40 \pm 0.03)$ $N \times\left(\log R_{\mathrm{SET}}\right)^{-1}$ and $(-3.48 \pm 0.26) N \times\left(\log R_{\mathrm{SET}}\right)^{-1}$ (both mean \pm fitting error) for $\mathrm{Chr} 21$ and $\mathrm{Chr} 18$, respectively.

This proposed method has better precision than the standard technique of working with differently labeled probes, which eliminates the problem of overspilling between fluorescent channels. However, this method is not capable of operating in the entire range of possible ratios of mixture components as the $R_{\mathrm{SET}}$ value has to be greater than the $R_{\mathrm{C}}$ to determine the $c n$ of the target without the FAM probe. Nevertheless, if the determination of the value with $R_{\mathrm{SET}}<R_{\mathrm{C}}$ is required, it is possible to change the system and use a FAM probe that is specific to the second target. The combination of the probe-based and intercalating dye-based methods brings the potential drawbacks of both such as the high cost for

Table 2. Information of Target Sequences and the Corresponding Primers and Probes

\begin{tabular}{|c|c|c|c|c|c|}
\hline target gene & gene location $^{a}$ & primer type & primer sequence $\left(5^{\prime}-3^{\prime}\right)$ & amplicon size (bp) & amplicon $T_{\mathrm{M}}\left({ }^{\circ} \mathrm{C}\right)$ \\
\hline \multirow[t]{3}{*}{ Chr21 } & $14099141-14099218$ & forward & ctaggagactgtccetgagctt & 78 & 83.5 \\
\hline & & reverse & agggggaacatagaggcttg & & \\
\hline & & LNA probe & FAM-ccctgcctct-BHQ1 & & \\
\hline \multirow[t]{4}{*}{ Chr18 } & $1248496-1248575$ & forward & ccatctccataacccaaatacc & 80 & 80.4 \\
\hline & & reverse & ccttgcaaacctcatgttga & & \\
\hline & & LNA probe & VIC-cccacctcca-BHQ1 & & \\
\hline & & & FAM-cccacctcca-BHQ1 & & \\
\hline
\end{tabular}

${ }^{a}$ Gene location according to the human genome assembly version hg19/Genome Reference Consortium Human Build 37. 
probe-based qPCR and the low specificity for intercalating dyebased qPCR. However, it is not necessary to have two expensive probes and a complex fluorescence system; thus, the proposed method can lower the cost as only one probe is applied. Additionally, it eliminates the fluorescence overspilling, which exists in probe-based qPCR multiplexing. Moreover, the melting curve analysis conducted immediately once the thermal cycling is completed will determine the amplicon specificity.

This proposed duplexing method using a single FITC fluorescent channel can be implemented for a wide variety of examinations where mixture analysis is desired once either multifluorescence channel is not a viable option or to lower the test cost. This qPCR duplexing thus opens new horizons for practical applications, providing an original, easily applicable technical solution.

\section{MATERIAL AND METHODS}

5.1. DNA Sequences and PCR Master Mix. We used the synthesized target fragments of Chr21 and Chr18 as well as primers and probes for both, as shown in Table 2. The chosen amplicons, designed primers, and the lock nucleic acids (LNA) probes were used beforehand for the noninvasive prenatal diagnostics using $\mathrm{dPCR}$, and they were optimized to perform in multiplex reactions. ${ }^{34} \mathrm{We}$ then prepared different types of PCR master mixes based on the following designed experiments by adjusting the volume of probes for both targets (Supporting Information, Section S6). The final volume of the PCR master mix was $\approx 10 \mu \mathrm{L}$ by adding sterilized deionized $\mathrm{H}_{2} \mathrm{O}$.

5.2. qPCR Protocol. We performed the qPCR with the protocol set in the following way: a hot start at $95{ }^{\circ} \mathrm{C}$ for $30 \mathrm{~s}$ to activate the polymerase followed by 40 cycles of denaturation at $95{ }^{\circ} \mathrm{C}$ for $10 \mathrm{~s}$, annealing at $52{ }^{\circ} \mathrm{C}$ for $30 \mathrm{~s}$, and elongation at $72{ }^{\circ} \mathrm{C}$ for $10 \mathrm{~s}$ using a commercial 96-well real-time PCR system collecting two $F$ values during each PCR cycle at the end of the elongation and denaturation steps. We then conducted an MCA from 65 to $95{ }^{\circ} \mathrm{C}$ with a ramping rate

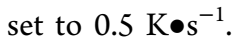

5.3. Data Processing. The collected data were processed using a MATLAB script by first splitting them into two blocks. The first 80 points contained the PCR amplification curves, and the rest represented the MCA. During each cycle, we collected two data points corresponding to the two adjacent sample points, $F$ values were collected at the end of the denaturation and elongation steps. Then, we split the amplification curve data according to their sequence. The data whose serial number was odd (even) were selected to form the denaturation (elongation) amplification curve. The elongation curve was the same as the conventional PCR amplification curve. The denaturation curve was different since the PCR cycle started with denaturation and ends with elongation. We skipped the first point of the denaturation curve as it had no meaning since no PCR amplification had occurred by that point in time. As a result, the denaturation curve had only 39 points, while the elongation had typical 40 points resulting from 40 cycles. After that, the amplification curves were processed independently.

5.4. Data Normalization. We normalized the amplification curves according to the following formula

$$
F_{\mathrm{N}}=\frac{F-F_{\text {min }}}{F_{\text {max }}-F_{\text {min }}}
$$

where $F_{\mathrm{N}}$ is the normalized $F$ amplitude value and $F_{\max }$ is the maximal amplitude value of the $F$ data. $F_{\min }$ is manually selected for each curve due to the unpredictable fluctuation in the $F$ value of the first few data. If the variation range $\left(F_{\max }-\right.$ $\left.F_{\text {min }}\right)$ of a curve is judged to be lower than 50000 arbitrary units (a.u.), this curve is regarded as a nonamplification curve, and the $F_{\mathrm{N}}$ value is set to 0 a.u.

5.5. $C_{t}$ Value Extraction. We performed the curve fitting using a Boltzmann function similar to the methods published earlier $^{35,36}$

$$
F_{\mathrm{N}}(i)=\frac{1}{1+\exp \left(\frac{i-i_{0}}{\mathrm{~d} F}\right)}+F_{\min } / F_{\max }
$$

where $F_{\mathrm{N}}(i)$ is the normalized amplitude of the $F$ value of cycle number $i, i_{0}$ is an inflexion point of the Boltzmann curve, and $\mathrm{d} F$ determines the Boltzmann curve slope at $F\left(i_{0}\right)$ by $\frac{\mathrm{d} F_{\mathrm{N}}(i)}{\mathrm{d} i}=-\frac{1}{4} \times \frac{F_{\max }}{\mathrm{d} F}$. In previous work, ${ }^{36}$ the author defined $C_{\mathrm{t}}$ as a value of $N$ at a fluorescence inflection point. Here, we calculated the $C_{t}$ value as an $N$ value for a $10 \%$ increase of the $F$ value above its baseline

$$
C_{\mathrm{t}}=F_{\text {min }}+0.1\left(F_{\text {max }}-F_{\text {min }}\right)
$$

This method is similar to the one conventionally used for calculating the $C_{\mathrm{t}}$ defining the $C_{\mathrm{t}}$ value as a cycle number for $10 \%$ of $F$ increased above the baseline. The Boltzmann function-based $C_{t}$ value determination was then implemented using the MATLAB script.

\section{ASSOCIATED CONTENT}

\section{Supporting Information}

The Supporting Information is available free of charge at https://pubs.acs.org/doi/10.1021/acsomega.1c02971.

Standard curves of single genes, amplicon ratios of Chr21 and Chr18 with Chr21 variable, MCAs between Chr21 with the FAM probe and Chr18 having $c n_{18}$ with three different values and $c n_{21}$ variable, amplicon ratios of Chr21 and Chr18 with Chr18 variable and MCA after PCR, amplicon ratios of Chr21 and Chr18, and PCR master mix (PDF)

\section{AUTHOR INFORMATION}

\section{Corresponding Author}

Pavel Neuzil - School of Mechanical Engineering, Department of Microsystem Engineering, Northwestern Polytechnical University, Xi'an, Shaanxi 710072, P. R. China; Central European Institute of Technology, Brno University of Technology, 61200 Brno, Czech Republic; School of Electrical Engineering and Computer Technology, Brno University of Technology, 61200 Brno, Czech Republic; ○ orcid.org/0000-0001-9040-281X; Email: pavel.neuzil@ nwpu.edu.cn

\section{Authors}

Haoqing Zhang - School of Mechanical Engineering, Department of Microsystem Engineering, Northwestern Polytechnical University, Xi'an, Shaanxi 710072, P. R. China

Zhiqiang Yan - School of Mechanical Engineering, Department of Microsystem Engineering, Northwestern Polytechnical University, Xi'an, Shaanxi 710072, P. R. China 
Xinlu Wang - School of Mechanical Engineering, Department of Microsystem Engineering, Northwestern Polytechnical University, Xi'an, Shaanxi 710072, P. R. China

Martina Gaňová - Central European Institute of Technology, Brno University of Technology, 61200 Brno, Czech Republic

Honglong Chang - School of Mechanical Engineering, Department of Microsystem Engineering, Northwestern Polytechnical University, Xi'an, Shaanxi 710072, P. R. China; (1) orcid.org/0000-0003-0400-3658

Soňa Lašsáková - Institute of Biology and Medical Genetics, First Faculty of Medicine, Charles University and General University Hospital in Prague, 12800 Prague, Czech Republic

Marie Korabecna - Institute of Biology and Medical Genetics, First Faculty of Medicine, Charles University and General University Hospital in Prague, 12800 Prague, Czech Republic

Complete contact information is available at:

https://pubs.acs.org/10.1021/acsomega.1c02971

\section{Notes}

The authors declare no competing financial interest.

\section{ACKNOWLEDGMENTS}

H.Z., Z.Y., X.W., and P.N. were supported by the Ministry of Science and Technology of the P.R. China [grant no. 2018YFE0109000]. M.K. and S.L. were supported by the Ministry of Education, Youth, and Sports of the Czech Republic [grant no. LTACH19005] and by a grant from the Ministry of Health of the Czech Republic [grant no. RVOVFN 64165].

\section{REFERENCES}

(1) Mullis, K.; Faloona, F.; Scharf, S.; Saiki, R.; Horn, G.; Erlich, H. Specific enzymatic amplification of DNA in vitro: the polymerase chain reaction. Cold Spring Harbor Symp. Quant. Biol. 1986, 263-273.

(2) Zhu, H.; Zhang, H.; Xu, Y.; Lašśáková, S.; Korabečná, M.; Neužil, P. PCR past, present and future. BioTechniques 2020, 69, 317-325.

(3) Shu, B.; Zhang, C.; Xing, D. Segmented continuous-flow multiplex polymerase chain reaction microfluidics for high-throughput and rapid foodborne pathogen detection. Anal. Chim. Acta 2014, 826, $51-60$.

(4) Kubista, M.; Andrade, J. M.; Bengtsson, M.; Forootan, A.; Jonák, J.; Lind, K.; Sindelka, R.; Sjöback, R.; Sjögreen, B.; Strömbom, L.; Ståhlberg, A.; Zoric, N. The real-time polymerase chain reaction. Mol. Aspects Med. 2006, 27, 95-125.

(5) Vogelstein, B.; Kinzler, K. W. Digital PCR. Proc. Natl. Acad. Sci. U.S.A. 1999, 96, 9236-9241.

(6) Zhu, H.; Fohlerová, Z.; Pekárek, J.; Basova, E.; Neužil, P. Recent advances in lab-on-a-chip technologies for viral diagnosis. Biosens. Bioelectron. 2020, 153, No. 112041.

(7) Shoute, L. C.; Loppnow, G. R. Characterization of the binding interactions between EvaGreen dye and dsDNA. Phys. Chem. Chem. Phys. 2018, 20, 4772-4780.

(8) Lehmusvuori, A.; Karhunen, U.; Tapio, A.-H.; Lamminmäki, U.; Soukka, T. High-performance closed-tube PCR based on switchable luminescence probes. Anal. Chim. Acta 2012, 731, 88-92.

(9) Zimmermann, B.; Holzgreve, W.; Wenzel, F.; Hahn, S. Novel real-time quantitative PCR test for trisomy 21. Clin. Chem. 2002, 48, $362-363$.

(10) Farzan, V. M.; Kvach, M. V.; Aparin, I. O.; Kireev, D. E.; Prikazchikova, T. A.; Ustinov, A. V.; Shmanai, V. V.; Shipulin, G. A.; Korshun, V. A.; Zatsepin, T. S. Novel homo Yin-Yang probes improve sensitivity in RT-qPCR detection of low copy HIV RNA. Talanta 2019, 194, 226-232.

(11) Chamberlain, J. S.; Gibbs, R. A.; Rainer, J. E.; Nguyen, P. N.; Thomas, C. Deletion screening of the Duchenne muscular dystrophy locus via multiplex DNA amplification. Nucleic Acids Res. 1988, 16, 11141-11156.

(12) Gaňová, M.; Zhang, H.; Zhu, H.; Korabečná, M.; Neužil, P. Multiplexed digital polymerase chain reaction as a powerful diagnostic tool. Biosens. Bioelectron. 2021, 181, No. 113155.

(13) DuVall, J. A.; Le Roux, D.; Thompson, B. L.; Birch, C.; Nelson, D. A.; Li, J.; Mills, D. L.; Tsuei, A.-c.; Ensenberger, M. G.; Sprecher, C.; Storts, D. R.; Root, B. E.; Landers, J. P. Rapid multiplex DNA amplification on an inexpensive microdevice for human identification via short tandem repeat analysis. Anal. Chim. Acta 2017, 980, 41-49.

(14) Li, Y.; Li, Y.; Zheng, B.; Qu, L.; Li, C. Determination of foodborne pathogenic bacteria by multiplex PCR-microchip capillary electrophoresis with genetic algorithm-support vector regression optimization. Anal. Chim. Acta 2009, 643, 100-107.

(15) Arnold, L.; Alexiadis, V.; watanaskul, T.; Zarrabi, V.; Poole, J.; Singh, V. Clinical validation of qPCR Target Selector assays using highly specific switch-blockers for rare mutation detection. J. Clin. Pathol. 2020, 73, 648-655.

(16) Emaus, M. N.; Anderson, J. L. Allelic discrimination between circulating tumor DNA fragments enabled by a multiplex-qPCR assay containing DNA-enriched magnetic ionic liquids. Anal. Chim. Acta 2020, 1124, 184-193.

(17) Muenchhoff, M.; Mairhofer, H.; Nitschko, H.; GrzimekKoschewa, N.; Hoffmann, D.; Berger, A.; Rabenau, H.; Widera, M.; Ackermann, N.; Konrad, R.; et al. Multicentre comparison of quantitative PCR-based assays to detect SARS-CoV-2, Germany, March 2020. Eurosurveillance 2020, 25, No. 2001057.

(18) Cottenet, G.; Blancpain, C.; Sonnard, V.; Chuah, P. F. Two FAST multiplex real-time PCR reactions to assess the presence of genetically modified organisms in food. Food Chem. 2019, 274, 760765 .

(19) Pazourkova, E.; Zednikova, I.; Korabecna, M.; Kralova, J.; Pisacka, M.; Novotna, M.; Calda, P.; Horinek, A. Optimization of diagnostic strategy for non-invasive cell-free foetal RHD determination from maternal plasma Vox Sang. 2021, 13099. DOI: 10.1111/ vox.13099

(20) Suwannakhon, N.; Pangeson, T.; Seeratanachot, T.; Mahingsa, K.; Pingyod, A.; Bumrungpakdee, W.; Sanguansermsri, T. Noninvasive prenatal screening test for compound heterozygous beta thalassemia using an amplification refractory mutation system realtime polymerase chain reaction technique. Hematol. Rep. 2019, 11, No. 8124

(21) Huggett, J. F.; The dMIQE Group; Whale, A. S.; et al. The digital MIQE guidelines update: minimum information for publication of quantitative digital PCR experiments for 2020. Clin. Chem. 2020, 66, 1012-1029.

(22) Bustin, S. A.; Benes, V.; Garson, J. A.; Hellemans, J.; Huggett, J.; Kubista, M.; Mueller, R.; Nolan, T.; Pfaffl, M. W.; Shipley, G. L.; et al. The MIQE guidelines: minimum information for publication of quantitative real-time PCR experiments. Clin. Chem. 2009, 55, 611622.

(23) Livak, K. J.; Schmittgen, T. D. Analysis of relative gene expression data using real-time quantitative PCR and the $2^{-\Delta \Delta \mathrm{CT}}$ method. Methods 2001, 25, 402-408.

(24) Gubala, A. J. Multiplex real-time PCR detection of Vibrio cholerae. J. Microbiol. Methods 2006, 65, 278-293.

(25) Waggoner, J. J.; Abeynayake, J.; Sahoo, M. K.; Gresh, L.; Tellez, Y.; Gonzalez, K.; Ballesteros, G.; Pierro, A. M.; Gaibani, P.; Guo, F. P.; et al. Single-reaction, multiplex, real-time rt-PCR for the detection, quantitation, and serotyping of dengue viruses. PLoS Neglected Trop. Dis. 2013, 7, No. e2116.

(26) Zhang, H.; Li, H.; Zhu, H.; Pekárek, J.; Podešva, P.; Chang, H.; Neužil, P. Revealing the secrets of PCR. Sens. Actuators, B 2019, 298, No. 126924. 
(27) Ahrberg, C. D.; Manz, A.; Neuzil, P. Single fluorescence channel-based multiplex detection of avian influenza virus by quantitative PCR with intercalating dye. Sci. Rep. 2015, 5, No. 11479.

(28) Zhu, H.; Zhang, H.; Ni, S.; Korabečná, M.; Yobas, L.; Neuzil, P. The vision of point-of-care PCR tests for the COVID-19 pandemic and beyond. TrAC, Trends Anal. Chem. 2020, 130, No. 115984.

(29) Ahrberg, C. D.; Neužil, P. Doubling throughput of a real-time PCR. Sci. Rep. 2015, 5, No. 12595.

(30) Rodriguez-Manzano, J.; Moniri, A.; Malpartida-Cardenas, K.; Dronavalli, J.; Davies, F.; Holmes, A.; Georgiou, P. Simultaneous single-channel multiplexing and quantification of carbapenemresistant genes using multidimensional standard curves. Anal. Chem. 2019, 91, 2013-2020.

(31) Moniri, A.; Rodriguez-Manzano, J.; Malpartida-Cardenas, K.; Yu, L.-S.; Didelot, X.; Holmes, A.; Georgiou, P. Framework for DNA quantification and outlier detection using multidimensional standard curves. Anal. Chem. 2019, 91, 7426-7434.

(32) Moniri, A.; Miglietta, L.; Malpartida-Cardenas, K.; Pennisi, I.; Cacho-Soblechero, M.; Moser, N.; Holmes, A.; Georgiou, P.; Rodriguez-Manzano, J. Amplification curve analysis: data-driven multiplexing using real-time digital PCR. Anal. Chem. 2020, 92, 13134-13143.

(33) Wang, C.; Gao, D.; Vaglenov, A.; Kaltenboeck, B. One-step real-time duplex reverse transcription PCRs simultaneously quantify analyte and housekeeping gene mRNAs. Biotechniques 2004, 36, 508519.

(34) Tan, C.; Chen, X.; Wang, F.; Wang, D.; Cao, Z.; Zhu, X.; Lu, C.; Yang, W.; Gao, N.; Gao, H. J. A.; et al. A multiplex droplet digital PCR assay for non-invasive prenatal testing of fetal aneuploidies. Analyst 2019, 144, 2239-2247.

(35) Liu, W.; Saint, D. A. Validation of a quantitative method for real time PCR kinetics. Biochem. Biophys. Res. Commun. 2002, 294, 347-353.

(36) Rutledge, R. Sigmoidal curve-fitting redefines quantitative realtime PCR with the prospective of developing automated highthroughput applications. Nucleic Acids Res. 2004, 32, No. e178. 